\title{
Validació inicial del JS NEO-A50, una versió abreviada del qüestionari de cinc factors de personalitat per a adolescents JS NEO-S
}

\author{
Jordi Ortet Walker \\ jortet@uji.es \\ Verónica Vidal Arenas \\ vvidal@uji.es \\ Francisco Javier López Fernández \\ frlopez@uji.es \\ Laura Mezquita Guillamón \\ Imezquit@uji.es \\ Manuel Ignacio Ibáñez Ribes \\ iribes@uji.es
}

\section{Resum}

L'adaptació espanyola per a adolescents del NEO PI-R, el JS NEO-S, és un instrument de 150 ítems que avalua el model de cinc factors de la personalitat. No obstant, per la població a la que es dirigeix aquest qüestionari, completar una escala de 150 ítems pot resultar costós. El nostre objectiu és desenvolupar una versió abreviada de 50 ítems del JS NEO-S. EI qüestionari es va administrar a una mostra de 400 participants d'entre 12 i 17 anys. Junt al JS NEO-S, també es va administrar un altre instrument de 5 factors, el BFPTSQ, així com el SENA i l'SLSS per avaluar símptomes psicopatològics i benestar subjectiu, respectivament. Finalment, es va dur a terme un retest d'un mes del JS A-50 i es van sol-licitar les notes. Es seleccionaren 50 ítems mitjançant anàlisi factorial exploratòria. Ítems de la majoria de facetes van estar representats en totes les dimensions. Els valors de validesa convergent presentaren correlacions d'entre 0,55 i 0,69 amb el BFPTSQ. Els valors de validesa de criteri també van ser adequats. Els índexs alfa per a les dimensions estigueren entre 0,73 i 0,83. Finalment, també es va trobar una fiabilitat d'estabilitat temporal adequada (d'entre 0,77 i 0,83 ). Aquests resultats evidencien unes propietats psicomètriques acceptables. Les limitacions principals del nostre treball serien, per una banda, la grandària mostral reduïda, així com la conveniència de replicar l'estudi amb una mostra independent. Futurs estudis haurien de continuar la validació del JS NEO-A50 atenent les limitacions indicades.

Paraules clau: personalitat, validació, psicometria, qüestionari, abreviat. 


\section{Abstract}

The Spanish adaptation of the NEO PI-R for adolescents, the JS NEO-S is a 150 -item instrument that assesses the Five-Factor Model of personality. However, for the population that this questionnaire is aimed at, completing a 150 -item scale can be complicated. Our aim is to develop an abridged, 50item version of the JS NEO-S. The questionnaire was administered to a sample of 400 participants between the ages of 12 and 17 . Along with the JS NEO-S, another 5-factor instrument was administered, the BFPTSQ, as well as the SENA and SLSS to assess psychopathological symptoms and subjective wellbeing, respectively. Finally, a one-month retest was conducted for the JS NEO-A50 and grades were requested. 50 items were selected employing an exploratory factor analysis. Items from the majority of the facets were represented in each of the dimensions. Convergent validity values yielded correlations between 0.55 and 0.69 with the BFPTSQ. Criterion validity values were also adequate. Alpha indices for the domains were between 0.73 and 0.83 . Finally, an adequate temporal stability reliability (between 0.77 and 0.83 ) was found. These results show acceptable psychometric properties. The main limitations of our study were, on the one hand, the small sample size, as well as the need for replication of the study with an independent sample. Future studies should continue to validate the JS NEO-A50 taking the highlighted limitations into account.

Keywords: personality, validation, psychometrics, questionnaire, abridged.

\section{Introducció}

La personalitat s'entén actualment com un constructe referit a les predisposicions de les persones cap a certes tendències emocionals, conductuals i cognitives. És una variable relativament estable en el temps i consistent en diferents contextos (Digman 1990). El model de l'estructura de la personalitat que té més suport en la actualitat és el model de cinc factors (MCF, o FFM en anglès) (McCrae 1991). Aquesta conceptualització proposa cinc dimensions bàsiques de personalitat: el neuroticisme, l'extraversió, l'obertura a l'experiència, l'amabilitat i la responsabilitat. Cada una d'estes dimensions tindria sis facetes de segon ordre cadascuna (30 en total).

S'han dut a terme nombrosos estudis relacionant certs trets de personalitat $i$ combinacions dels mateixos, amb diversos àmbits al llarg del cicle vital com la salut física i psicològica, l'èxit acadèmic i laboral o les conductes delictives (Hampson, Goldberg, Volgt i Dubanoski 2006; Kubicka, Matejcek, Dytrych i Roth 2001; Shiner, Masten i Roberts 2003). L'associació entre les característiques de personalitat i els trastorns mentals té un gran interés entre la comunitat científica i s'han identificat els trets de baixa extraversió (emocions positives reduïdes) i alt neuroticisme (emocions negatives incrementades) com a importants correlats de transtorns de l'espectre internalitzant com la depressió i l'ansietat (Mezquita et al. 2015). Tanmateix, les conductes externalitzants, com ara les agressions o el comportament antisocial, s'han associat principalment amb els trets de baixa amabilitat (antagonisme) i baixa responsabilitat (desinhibició) (Sleep, Hyatt, Lamkin, Maples-Keller i Miller 2018). El perfil de personalitat més relacionat amb l'èxit acadèmic seria l'alta responsabilitat. Un estudi recent de Rimfeld, Kovacs, Dale i Plomin (2016) identifica aquesta dimensió com el principal tret de personalitat predictor de bons resultats acadèmics en estudiants d'institut britànics. 
Quant a la satisfacció amb la vida, aquesta és una variable que s'ha trobat fortament lligada al tret d'estabilitat emocional, on aquells participants menys neuròtics mostraven una major satisfacció vital, en un estudi recent de Marcionetti i Rossier (2016).

L'instrument d'avaluació del MCF més emprat és el NEO PI-R (McCrae i Costa 2010), un qüestionari de 240 ítems que avalua tant les cinc grans dimensions de personalitat com les seues corresponents facetes. És una escala de tipus Likert de 5 punts $(0=$ Totalment en desacord; $4=$ Totalment d'acord $)$. Completar un qüestionari d'aquesta extensió pot ser complicat en administracions de bateries amples de tests, per la qual cosa s'han desenvolupat formes curtes, com el NEO-FFI (McCrae i Costa 2010). Els instruments curts tenen propietats psicomètriques adequades i són més fàcils d'administrar i corregir que les seues versions llargues.

En el nostre entorn sociocultural, un qüestionari curt dirigit a adolescents de parla castellana basat en el MCF és el JS NEO-S (Ortet et al. 2012) que consta de 150 ítems i avalua la personalitat tant a nivell de dimensions com de facetes. Tot $\mathrm{i}$ l'extensió reduïda respecte al NEO PI-R, el desenvolupament d'un qüestionari encara més curt per a avaluar el MCF a nivell de les dimensions seria de gran utilitat per als contextos de recerca en els que s'han d'avaluar diversos constructes.

Per aquestes raons, l'objectiu d'aquest estudi és desenvolupar un instrument abreviat del JS NEO-S de 50 ítems (10 ítems per dimensió).

Les hipòtesis principals serien dues:

1) L'estructura del qüestionari abreviat representarà els cinc factors de forma adequada, mostrant un índex acceptable de consistència interna dels mateixos.

2) Les propietats psicomètriques de fiabilitat d'estabilitat temporal, validesa de criteri i validesa convergent seran adequades i reflectiran els correlats de personalitat que es troben habitualment a la literatura.

\section{Mètode}

\section{Participants i procediment}

L'estudi es va dur a terme amb una mostra total de $n=400$ estudiants d'institut d'entre 12 i 17 anys de Castelló de la Plana (Mitjana d'edat=14,31; DT=1,57; $51 \%$ homes, $49 \%$ dones). Es van fer anar submostres per a les proves de validesa de criteri $(n=378)$, validesa convergent $(n=368)$ i per a dur a terme un retest del JS NEO-A50 $(n=152)$.

La mostra consistia en adolescents dels instituts que va contactar el nostre grup de recerca. Els centres van accedir a permetre'ns recopilar les dades en les hores de tutories dels alumnes (una hora a la setmana). D'aquesta manera, el mostreig es va dur a terme a nivell de grup, conformant els instituts sencers i obtenint així una mostra representativa d'una cohort d'adolescents. Es van requerir tres sessions d'una hora per a que cada grup completés tots els qüestionaris. Una quarta sessió s'emprà per a dur a terme retests d'algunes de les mesures, incloent el JS NEO-A50, així com acabar els qüestionaris incomplets de sessions anteriors.

La participació a l'estudi va ser completament voluntària. Es van repartir incentius a cadascun dels participants per a tractar d'incrementar la participació, consistint en 
productes de marxandatge de la nostra universitat com llibretes i bolígrafs. S'encarregaren d'administrar i recollir les dades alguns estudiants de grau i postgrau, què aprofitaren les dades per a dur a terme els seus treballs de fi de grau o màster.

La majoria de la mostra eren persones menors d'edat, per la qual cosa es repartiren fulls de consentiment informat per a que signaren els pares o tutors, d'acord amb la Llei Europea de Protecció de Dades (European Union General Protection Regulation 2016/679). Tanmateix, tota aquella informació personal es va tractar amb la màxima confidencialitat.

\section{Instruments}

Els instruments que es van utilitzar per al present estudi van ser els següents:

- La versió espanyola junior del NEO PI-R (JS NEO-S) (Ortet et al. 2012) és la forma curta de l'adaptació espanyola del qüestionari de personalitat NEO PI-R per a adolescents d'edats compreses entre els 12 i 17 anys. Es tracta d'una escala de 150 ítems de tipus Likert, amb 5 punts de resposta $(0=$ Totalment en desacord; 4=Totalment d'acord). És un qüestionari que avalua les 5 dimensions del MCF (neuroticisme, extraversió, obertura, amabilitat i responsabilitat), així com les 30 facetes corresponents a cada una d'elles (6 facetes per dimensió). Les propietats psicomètriques del qüestionari són adequades i poden consultar-se a: Ortet et al. 2012. Es va emprar aquesta escala com a base de dades secundària de la que extraure els 50 ítems per al JS NEO-A50, utilitzant una anàlisi factorial exploratòria (EFA) i avaluant l'adequació dels ítems segons les saturacions en les seues dimensions corresponents. Tanmateix, s'observaren els valors de consistència interna per a cada dimensió una vegada es seleccionaren els ítems per a la versió abreviada.

- El Big Five Personality Questionnaire (BFPTSQ) (Morizot 2014) és un qüestionari de personalitat curt de 50 ítems que serveix per a avaluar les dimensions del MCF. Està dirigit a la població adolescent i té unes propietats psicomètriques adequades que poden trobar-se a: Morizot 2014. S'emprà aquesta escala per a avaluar la validesa convergent per al JS NEO-A50, degut a que les dimensions entre ambdós instruments són anàlogues.

- El Sistema de Evaluación para Niños y Adolescentes (SENA) (SánchezSánchez, Fernández-Pinto, Santamaría, Carrasco i del Barrio 2016) és un qüestionari de 114 ítems que avalua problemes emocionals i conductuals de xiquets i adolescents d'entre 3 i 18 anys. Té unes propietats psicomètriques adequades que poden trobar-se a: Sánchez-Sánchez et al. 2016. Es van seleccionar quatre escales d'aquest instrument, dues corresponents a l'espectre de conducta externalitzant (conductes agressives i antisocials) i dues de problemes internalitzants (problemes ansiosos i depressius). Aquestes variables es triaren per a avaluar la validesa de criteri del nostre qüestionari abreviat, degut a la relació entre certes combinacions de trets de personalitat $\mathrm{i}$ aquests correlats conductuals i emocionals trobats a la literatura (Krueguer 1999; Sleep, Hyatt, Lamkin, Maples-Keller i Miller 2018).

- El Student's Life Satisfaction Scale (SLSS) (Huebner, Laughlin, Ash i Gilman 1998) és una escala breu de 7 ítems que avalua la satisfacció amb la vida autoinformada per a xiquets i adolescents d'entre 8 i 18 anys. Conté 5 subescales corresponents a la satisfacció en diversos dominis: la família, els amics, l'escola, la llar i el self. Les propietats psicomètriques del qüestionari són adequades i poden trobar-se a: Huebner, Laughlin, Ash i Gilman 1998. S'emprà l'escala per a avaluar la validesa de criteri, degut a que s'ha trobat a la 
literatura una relació clara entre satisfacció amb la vida i certes combinacions de trets de personalitat (Marcionetti i Rossier 2016).

\section{Anàlisis}

L'anàlisi de dades va consistir, en primer lloc, en la construcció de la versió abreviada del JS NEO-S. L'estratègia que es va seguir és l'habitual per a construir versions curtes (Widaman, Little, Preacher i Sawalani 2011). Aquest procediment instrumental involucra dues fases. La primera consisteix en dur a terme un EFA per a cada dimensió per separat en la base de dades de la versió de 150 ítems, seleccionant així els ítems amb les saturacions més altes. En segon lloc, els 50 ítems seleccionats en blocs de 10 es van sotmetre a un EFA en conjunt i a una anàlisi de consistència interna, mitjançant l'alfa de Cronbach, per a cada dimensió de 10 ítems per separat. Tenint en compte ambdúes mesures, els ítems es seleccionaren i substituiren de forma instrumental de l'anàlisi factorial original de 50 ítems per a tenir un bon equilibri entre la representació de les facetes i la consistència interna de les dimensions de la nova escala de 50 ítems.

Quan l'escala abreviada tingué una estructura i consistències internes adequades, es van dur a terme més anàlisis de dades per a obtindre altres propietats psicomètriques. La fiabilitat d'estabilitat temporal es va analitzar mitjançant dades del retest d'un mes. La validesa convergent es va obtindre amb les correlacions entre les dimensions del JS NEO-A50 i les del BFPTSQ. La validesa de criteri es va extraure amb les correlacions entre les dimensions del JS NEO-A50 i les variables de problemes externalitzants i internalitzants (de l'escala SENA), la satisfacció amb la vida (escala SLSS) i els resultats acadèmics (les notes sol-licitades).

Totes les anàlisis estadístiques es van dur a terme amb el programari SPSS, versió 24.

\section{Resultats}

L'EFA per a l'escala sencera va mostrar unes saturacions adequades $(\geq 0,30)$ per als ítems seleccionats en les seues respectives dimensions. No van poder estar-hi representades les següents facetes degut a les saturacions insuficients dels ítems de les mateixes: impulsivitat, de la dimensió de neuroticisme; accions i valors, de la dimensió d'obertura; i confiança, de la dimensió d'amabilitat. Així, 26 de les 30 facetes del JS NEO-S tingueren almenys un ítem que els representara. Tanmatiex, les facetes que es van incloure mostraren unes saturacions dels seus ítems $\geq 0,30$ només per a les dimensions a les que pertanyen. 9 de les facetes comptaren amb 1 ítem cadascuna, 10 facetes tingueren 2 ítems, 6 facetes estaven representades per 3 ítems i, finalment, 1 de les facetes comptava amb 4 ítems (vegeu la taula 1). 
Taula 1

Anàlisi Factorial Exploratori d'eixos principals del JS NEO-S

\begin{tabular}{|c|c|c|c|c|c|}
\hline \multirow[t]{2}{*}{ Ítems JS NEO-S (facetes) } & \multicolumn{5}{|c|}{ Dimensions } \\
\hline & $\mathrm{N}$ & $E$ & $\mathrm{O}$ & $A$ & C \\
\hline 88 (vulnerabilitat) & 0,71 & & & & \\
\hline 134 (depressió) & 0,69 & & & & \\
\hline 109 (ansietat social) & 0,67 & & & & \\
\hline 103 (depressió) & 0,61 & & & & \\
\hline 47 (ansietat social) & 0,57 & & & & \\
\hline 129 (hostilitat) & 0,52 & & & & \\
\hline 124 (ansietat) & 0,52 & & & & \\
\hline 98 (hostilitat) & 0,46 & & & 0,29 & \\
\hline 26 (vulnerabilitat) & 0,46 & & & & \\
\hline 16 (ansietat social) & 0,42 & & & & \\
\hline 79 (activitat) & & 0,75 & & & \\
\hline 120 (emocions positives) & & 0,69 & & & \\
\hline 63 (cordialitat) & & 0,61 & & & \\
\hline 58 (emocions positives) & & 0,59 & & & \\
\hline 151 (emocions positives) & & 0,52 & & & \\
\hline 94 (cordialitat) & & 0,48 & & & \\
\hline 38 (gregarisme) & & 0,48 & & & \\
\hline 17 (activitat) & & 0,45 & & & \\
\hline 135 (asertivitat) & & 0,43 & & & \\
\hline 84 (recerca d'emocions) & & 0,32 & & 0,26 & \\
\hline 69 (estètica) & & & 0,63 & & \\
\hline 131 (estètica) & & & 0,62 & & \\
\hline 85 (ideas) & & & 0,52 & & \\
\hline 33 (fantasía) & 0,28 & & 0,47 & & 0,25 \\
\hline 23 (idees) & & & 0,44 & & \\
\hline 147 (idees) & & & 0,42 & & \\
\hline 95 (fantasia) & & & 0,40 & & \\
\hline 8 (estètica) & & & 0,37 & & \\
\hline 136 (sentiments) & 0,27 & 0,28 & 0,34 & & \\
\hline 100 (estètica) & & & 0,30 & & \\
\hline 101 (rranquesa) & & & & 0,66 & \\
\hline 9 (franquesa) & & & & 0,64 & \\
\hline
\end{tabular}




\begin{tabular}{|c|c|c|c|}
\hline 132 (franquesa) & & 0,62 & \\
\hline 76 (altruisme) & & 0,60 & \\
\hline 45 (altruisme) & & 0,56 & \\
\hline 86 (modèstia) & & 0,52 & \\
\hline 14 (altruisme) & & 0,51 & \\
\hline 153 (sensibilitat als demés) & & 0,48 & \\
\hline 148 (modèstia) & & 0,48 & \\
\hline 19 (actitud conciliadora) & & 0,40 & \\
\hline 25 (autodisciplina) & & & 0,65 \\
\hline 108 (sentit del deure) & & 0,29 & 0,62 \\
\hline 102 (ordre) & 0,29 & & 0,59 \\
\hline 82 (necesitat d'assoliment) & & & 0,58 \\
\hline 113 (necesidad d'assoliment) & & & 0,55 \\
\hline 66 (competència) & & & 0,52 \\
\hline 10 (ordre) & & & 0,50 \\
\hline 36 (ordre) & & & 0,48 \\
\hline 87 (autodisciplina) & & 0,25 & 0,48 \\
\hline 92 (deliberació) & & & 0,44 \\
\hline
\end{tabular}

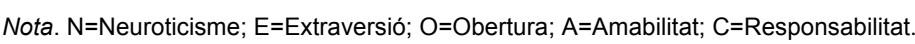

Rotació Varimax. No es mostren les saturacions inferiors a 0,25 . Les saturacions dels ítems en les dimensions corresponents es mostren en negreta. Totes les saturacions es mostren en valors absoluts.

Es van trobar uns valors acceptables de consistència interna i fiabilitat temporal. Cadascuna de les dimensions tingué una consistència interna adequada (Nunnally 1978). Les correlacions entre les dimensions avaluades a la sessió inicial i les mateixes transcorregut un mes eren altes i significatives per a tots cinc trets (vegeu la taula 2).

Quant a la validesa convergent, les correlacions més altes i significatives es van trobar entre dimensions de personalitat anàlogues. Totes tenien la mateixa nomenclatura excepte el neuroticisme del JS NEO-A50, corresponent a la dimensió d'estabilitat emocional (en el pol positiu) del BFPTSQ (vegeu la taula 3).

Per últim, l'anàlisi de validesa de criteri va aportar les correlacions esperades entre les dimensions de personalitat i les variables externes avaluades. Els problemes de conducta agressius i antisocials del factor externalitzant es trobaren significativament associades amb les puntuacions més baixes en amabilitat i responsabilitat. Els problemes d'ansietat i depressió es relacionaren significativament amb les puntuacions altes en neuroticisme, mentre que la satisfacció amb la vida estigué correlacionada significativament amb puntuacions baixes d'aquesta dimensió. Els resultats acadèmics estaven correlacionats significativament amb les puntuacions altes en la dimensió de responsabilitat. Es trobaren altres correlacions significatives, més baixes, entre els trets de personalitat amb aquestes mesures: els problemes antisocials i aggressius correlacionaren negativament amb l'obertura. Els problemes depressius s'associaren negativament amb l'extraversió, l'amabilitat i la responsabilitat, patró que es va repetir per als problemes ansiosos per a les dues últimes dimensions. La satisfacció amb la vida va correlacionar positivament amb l'extraversió, l'amabilitat i la responsabilitat. Per 
últim, el rendiment acadèmic estava positivament associat amb l'obertura i l'amabilitat (vegeu la taula 4).

Taula 2

Fiabilitat i consistència interna i d'estabilitat temporal del JS NEO-A50

\begin{tabular}{lll}
\hline Dimensions & Alfa & Retest $^{(1)}$ \\
\hline Neuroticisme & 0,83 & $0,77^{* * \star}$ \\
Extraversió & 0,79 & $0,76^{* * \star}$ \\
Obertura & 0,73 & $0,79^{* \star *}$ \\
Amabilitat & 0,82 & $0,78^{* \star *}$ \\
Responsabilitat & 0,83 & $0,83^{* * *}$ \\
\hline
\end{tabular}

Taula 3

Validesa Convergent

\begin{tabular}{llllll}
\hline Dimensions & BFPTSQ & BFPTSQ & BFPTSQ & BFPTSQ & BFPTSQ \\
& Em. St. & E & O & A & C \\
\hline JS NEO-A50 N & $\mathbf{- 0 , 5 7}^{* * *}$ & $-0,26^{* * *}$ & $0,11^{* *}$ & $0,26^{* * *}$ & $-0,29^{* * *}$ \\
JS NEO-A50 E & 0,01 & $\mathbf{0 , 5 8}^{* * *}$ & $0,19^{* * *}$ & $-0,20^{* * *}$ & 0,07 \\
JS NEO-A50 O & $-0,20^{* * *}$ & 0,02 & $\mathbf{0 , 6 1}$ & $-0,11^{* *}$ & 0,09 \\
JS NEO-A50 A & $0,15^{* *}$ & $-0,01$ & $-0,02$ & $\mathbf{0 , 5 5}^{* * *}$ & $0,33^{* * *}$ \\
JS NEO-A50 C & $0,12^{*}$ & $0,15^{* *}$ & $0,12^{*}$ & $-0,38^{* * *}$ & $\mathbf{0 , 6 9}$ \\
\hline
\end{tabular}

Nota. $\mathrm{N}=$ Neuroticisme; $\mathrm{E}=$ Extraversió; $\mathrm{O}=$ Obertura; $\mathrm{A}=\mathrm{Amabilitat} ; \mathrm{C}=\mathrm{Responsabilitat}$. ${ }^{*} p<0,05$. ${ }^{* *} p<0,01$. ${ }^{* *} p<0,001$. $\mathrm{n}=378$.

Taula 4

Validesa de criteri

\begin{tabular}{|c|c|c|c|c|c|c|}
\hline $\begin{array}{l}\text { Dimensions } \\
\text { JS NEO-A50 }\end{array}$ & Antisocial & Agressivitat & Ansietat & Depressió & $\begin{array}{l}\text { Satisfacció } \\
\text { amb la vida }\end{array}$ & Notes \\
\hline $\mathrm{N}$ & $0,15^{\star *}$ & $0,17^{* *}$ & $0,60^{* * *}$ & $0,64^{* \star *}$ & $-0,38^{* * *}$ & $-0,05$ \\
\hline$E$ & $0,14^{* *}$ & 0,09 & 0,04 & $-0,19^{* * *}$ & $0,25^{* * *}$ & $-0,01$ \\
\hline 0 & $-0,11^{*}$ & $-0,17^{\star *}$ & $0,27^{\star \star *}$ & $0,22^{* \star *}$ & $-0,05$ & $0,26^{\star \star \star}$ \\
\hline$A$ & $-0,44^{* \star *}$ & $-0,50^{* \star *}$ & $-0,08$ & $-0,18^{* * *}$ & $0,18^{* * *}$ & $0,20^{\star \star \star}$ \\
\hline C & $-0,37^{* \star *}$ & $-0,32^{* * *}$ & $-0,16^{* *}$ & $-0,23^{* * *}$ & $0,22^{* * *}$ & $0,41^{* \star \star}$ \\
\hline
\end{tabular}

Nota. $\mathrm{N}=$ Neuroticisme; $\mathrm{E}=$ Extraversió; $\mathrm{O}=\mathrm{Obertura}$; $\mathrm{A}=\mathrm{Amabilitat}$; $\mathrm{C}=$ Responsabilitat; Em. St.=Estabilitat emocional. Les correlacions entre dimensions corresponents es mostren en negreta. ${ }^{*} p<0,05 .{ }^{* *} p<0,01 .{ }^{* * *} p<0,001$.

$\mathrm{n}=368$.

\section{Discussió i conclusions}

Els resultats mostren l'estructura i les propietats psicomètriques adequades de l'escala abreviada que s'ha desenvolupat, el JS NEO-A50. Així, les hipòtesis plantejades s'han confirmat i podem concloure que aquest qüestionari és un instrument útil per a l'avaluació de la personalitat en adolescents de parla castellana.

Per una banda, d'acord amb la primera hipòtesi, les saturacions factorials obtingudes dels ítems sobre les seues respectives dimensions (i no sobre aquelles no 
corresponents) validarien l'estructura del questionari. Junt a aquests índexs obtinguts mitjançant l'EFA, les anàlisis de consistència interna per a cada una de les dimensions tingueren valors adequats. L'objectiu de l'estudi era obtindre un questionari abreviat que puguera avaluar les dimensions de personalitat del MCF, però voliem obtindre la representació més alta possible de les 30 facetes. No obstant la dificultat d'obtindre aquest resultat degut a l'extensió reduïda de l'intrument, només foren quatre les facetes no reflectides al JS NEO-A50. La dimensió més costosa de respresentar fou l'obertura, consistent amb estudis a la literatura què indiquen que aquest tret potser no es troba completament desenvolupat en els adolescents o els seus ítems tan ben compresos com en les avaluacions d'adults.

Respecte a la segona hipòtesi, el JS NEO-A50 mostra uns valors de fiabilitat temporal, validesa convergent i validesa de criteri adequats. Una limitació important va ser la mort experimental que vam sofrir a l'hora de dur a terme el retest (menys de la meitat de la mostra total va completar-lo). Tot i així, les correlacions entre les dimensions d'ambdúes sessions d'administració van ser altes. Les correlacions entre les dimensions anàlogues del JS NEO-A50 amb les del BFPTSQ també resultaren acceptables i en la línia esperada, i les correlacions més altes van ser aquelles entre dimensions corresponents. En la mateixa línia que els estudis de Sleep, Hyatt, Lamkin, Maples-Keller i Miller (2018), Marcionetti i Rossier (2016) i Rimfeld, Kovacs, Dale i Plomin (2016), les dimensions de personalitat més relacionades amb els problemes externalitzants i internalitzants, satisfacció amb la vida i rendiment acadèmic es van replicar. Quant a les correlacions secundàries per a la validesa de criteri, la troballa indicant que els participants més oberts i amables obtenen millors resultats acadèmics està en la mateixa línia que altres estudis a la literatura (Noftle i Robins 2007; Vedel i Poropat 2017) on aquests trets de personalitat són el segon i tercer més rellevants a I'hora de predir els resultats acadèmics, respectivament, després de la responsabilitat. Les correlacions secundàries trobades per als problemes internalitzants i externalitzants es troben també en estudis com la meta-anàlisi de Kotov et al. (2010), amb un èmfasi particular en la relació entre la baixa responsabilitat i els síntomes internalitzants.

Les limitacions principals de l'estudi serien, per una banda, la mort experimental ja discutida, així com el tamany redüit de la mostra.

En definitiva, s'han de dur a terme més estudis amb mostres més amples per a continuar validant el JS NEO-A50, on hauria d'incloure's una anàlisi factorial confirmatòria (CFA) amb una mostra independent.

\section{Referències bibliogràfiques}

European Parliament. 2016. Regulation (EU) 2016/679 of the European Parliament and of the council. Recuperat de: https://eur-lex.europa.eu/eli/reg/2016/679/oj [Consultat el 15 de maig de 2019].

Digman, John M. 1990. «Personality structure: Emergence of the five-factor model». Annual Review of Psychology, 41: 417-440.

Hampson, Sarah E., Lewis R. Goldberg, Thomas M. Vogt i Joan P. Dubanoski. 2006. «Forty years on: Teachers' assessments of children's personality traits predict self-reported health behaviors and outcomes at midlife». Health Psychology, 25: 57-64.

Huebner, E. Scott, James E. Laughlin, Chris Ash i Rich Gilman. 1998. «Further validation of the Multidimensional Students' Life Satisfaction Scale». Journal of Psychoeducational Assessment, 16: 118-134. 
Kotov, Roman et al. 2017. "The Hierarchical Taxonomy of Psychopathology: A dimensional alternative to traditional nosologies». Journal of Abnormal Psychology, 126: 454-477.

Krueger, Robert F. 1999. "The structure of common mental disorders». Archives of General Psychiatry, 56: 921-926.

Kubicka, Ludek, Zdenek Matejcek, Zdenek Dytrych i Zdenek Roth. 2001. «IQ and personality traits assessed in childhood as predictors of drinking and smoking behaviour in middle-aged adults: A 24-year follow-up study». Addiction, 96: 1615-1628.

Marcionetti, Jenny i Jerôme Rossier. 2016. «Global life satisfaction in adolescence: The role of personality traits, self-esteem, and self-efficacy». Journal of Individual Differences, 37: 135-144.

McCrae, Robert R. 1991. "The five-factor model and its assessment in clinical settings». Journal of Personality Assessment, 57: 399-414.

McCrae, Robert R. i Paul T Costa. 2010. NEO Inventories for the NEO Personality Inventory-3 (NEO-PI-3), NEO Five-Factor Inventory-3 (NEO-FFI-3), NEO Personality Inventory-Revised (NEO-PI-R): Professional manual. Lutz, FL: Psychological Assessment Resources.

Mezquita, Laura, Manuel Ignacio Ibáñez, Helena Villa, Lourdes Fañanás, Jorge MoyaHigueras i Generós Ortet. 2015. «Five-factor model and internalizing and externalizing syndromes: A 5-year prospective study». Personality and Individual Differences, 79: 98-103.

Morizot, Julien. 2014. "Construct validity of adolescents' self- reported big five personality traits: Importance of conceptual breadth and initial validation of a short measure». Assessment, 21: 580-606.

Noftle, Erik E. i Richard W. Robins. 2007. «Personality predictors of academic outcomes: big five correlates of GPA and SAT scores». Journal of Personality and Social Psychology, 93: 116-130.

Nunnally, Jum C. 1978. Psychometric theory ( $2^{\text {nd }}$ ed.). New York: McGraw-Hill.

Ortet Generós, Manuel, Ignacio Ibáñez, Jorge Moya, Helena Villa, Ana Viruela i Laura Mezquita. 2012. "Assessing the five factors of personality in adolescents: The junior version of the Spanish NEO-PI-R». Assessment, 19: 114-130.

Rimfeld, Kaili, Yulia Kovas, Philip S. Dale i Robert Plomin. 2016. "True grit and genetics: Predicting academic achievement from personality». Journal of Personality and Social Psychology, 111: 780-789.

Sánchez-Sánchez, Fernando, Irene Fernández-Pinto, Pablo Santamaría, Miguel A. Carrasco i Victoria del Barrio. 2016. «SENA, Sistema de Evaluación de Niños y Adolescentes: proceso de desarrollo y evidencias de fiabilidad y validez». Revista de Psicología Clínica con Niños y Adolescentes, 3: 23-34.

Shiner, Rebecca L., Ann S. Masten i Jennifer M. Roberts. 2003. «Childhood personality foreshadows adult personality and life outcomes two decades later». Journal of Personality, 71: 1145-1170.

Sleep, Chelsea E., Courtland S. Hyatt, Joanna Lamkin, Jessica L. Maples-Keller i Joshua D. Miller. 2018. «Examining the relations among the DSM-5 alternative model of personality, the five-factor model, and externalizing and internalizing behavior». Personality Disorders: Theory, Research, and Treatment, 9: 379384.

Vedel, Anna i Arthur Poropat. 2017. "Personality and Academic Performance». En Encyclopedia of Personality and Individual Differences, eds. Virgil Zeigler-Hill i Todd K. Shackelford. New York: Springer.

Widaman, Keith F., Todd D. Little, Kristopher J. Preacher i Gita M. Sawalani. 2011. "On creating and using short forms of scales in secondary research». En Secondary data analysis: An introduction for psychologists, eds. Trzesniewski, Kali H., M. Brent Donnellan i Richard E. Lucas. Washington, DC: American Psychological Association. 